\title{
Therapeutic Applications of Vitamin D Supplementation on Type 2 Diabetes
}

\author{
Louay Labban \\ Department of Nutrition and Food Sciences, Faculty of Health Sciences, University of Kalamoon, Deir Atiyah, \\ Syria \\ Email: drlouay@gmail.com
}

Received 15 March 2014; revised 20 April 2014; accepted 3 May 2014

Copyright (C) 2014 by author and OALib.

This work is licensed under the Creative Commons Attribution International License (CC BY).

http://creativecommons.org/licenses/by/4.0/

(c) (i) Open Access

\section{Abstract}

Background: Many studies have found that vitamin D deficiency is associated with impaired human insulin action, suggesting a role in the pathogenesis of diabetes mellitus type 2 (T2DM). This study investigated the effects of vitamin D3 supplementation on serum HbA1c values and 25(OH) vitamin $D$ concentrations in healthy and diabetic individuals. Methods: $\mathbf{7 2 2}$ individuals aged from 36 to 61 years old were selected (360 males and 362 females) divided into two groups. Healthy male group consisted of 180 person with HbA1c value $(6.78 \pm 2.14)$ and 180 persons diagnosed with type 2 diabetes $(\mathrm{HbA1c} 11.64 \pm 3.85)$. The healthy female group had also 180 person (HbA1c $7.42 \pm 2.86$ ) and 182 diabetic females (HbA1c $11.43 \pm 2.88$ ). Each group was then divided into two subgroups. One supplemented with (2000 IU/day) of vitamin $D$ for 90 days and the other one was not given any vitamin D supplements. HbA1c and serum 25(OH) vitamin D were recorded. Results: Mean serum HbA1c values for both males and females improved with vitamin $D$ supplementations but the improvement was significant in the diabetic group (males and females) comparing with healthy group. Serum HbA1c in healthy group improved by $4.8 \%$ and $2.7 \%$ for males and females respectively whereas serum HbA1c for diabetic group declined by $13.4 \%$ and $21.8 \%$ for group 7 9 and 9.6 and $22.1 \%$ for group $>9$ for males and females respectively. Also, serum 25(OH) vitamin D concentrations improved dramatically with supplementation in healthy and diabetic groups. 25(OH) vitamin $D$ for the healthy males improved by $67.9 \%$ and for healthy females $47.7 \%$ whereas for diabetic males and females improved by $53 \%$ and $31.7 \%$ and $56.6 \%$ and $77.2 \%$ for males and females HbA1c 7 - 9 and for HbA1c $>9$ respectively. Conclusion: Vitamin D supplementation lowered serum HbA1c values of males and females especially in the group Hba1c values $>9$ and this can be considered as a cheap and effective therapeutic tool in the management of type 2 diabetes. Also vitamin D supplementation improved concentration of serum $25(\mathrm{OH})$ vitamin $D$ in both healthy and diabetic group upgrading their vitamin D status from deficiency to sufficiency. 
Keywords

Vitamin D Supplements, Diabetes Type 2, HbA1c, 25(OH) Vitamin D, Syrian

\section{Introduction}

Diabetes Mellitus is considered a major chronic disease and its complications have become a main cause of morbidity and mortality worldwide. Prevalence of type 2 diabetes mellitus (T2DM) is increasing worldwide. It is estimated that over 360 million people are suffering from diabetes and it is expected that its prevalence reach a staggering 552 million by 2030 [1]. T2DM is defined by impaired glucose tolerance, chronic hyperglycemia, altered insulin secretion, and complications that come from induction of oxidative stress [2]. One of novel strategy toward prevention and control of T2DM is vitamin D supplementation.

Vitamin D deficiency is also considered a public health problem around the world. In 2008, it was estimated that 1 billion individuals presented vitamin D insufficiency or deficiency [3]. Vitamin D deficiency is generally due to a lack of adequate sunlight exposure or excessive use of sunscreen, and/or inadequate nutritional vitamin D intake [4]. Vitamin D is an essential element for establishing and maintaining bone structure. Vitamin D deficiency results in rickets and osteomalacia. Even slight vitamin D deficiency results in secondary hyperparathyroidism and increased bone resorption [5] [6]. In addition, there has been increased attention to the physiologic importance of vitamin D in non-skeletal tissues [7].

Vitamin D is supplied by consumption of vitamin D-rich foods and by vitamin D synthesis in skin. Natural nutrient materials are not a sufficient source of vitamin $\mathrm{D}$ to supply the body requirements; therefore where there is no supplementation of foodstuffs, the main source for vitamin D is produced by UV light [8] [9].

The extreme consequences of vitamin D deficiency such as rickets in children and osteomalacia in adults have been almost eliminated in some developed countries through adequate diet, food fortification, and the encouragement of moderate sunlight exposure [10].

There are many studies suggested that vitamin D plays a major role in several mechanisms in addition to bone metabolism [11] and is involved in abnormal glucose metabolism as well as in type 2 diabetes [12]. A recent review indicates that vitamin D deficiency may predispose to glucose intolerance, altered insulin secretion and type 2 diabetes [13], either through a direct action via vitamin D receptor (VDR) activation or indirectly via calcemic hormones and also via inflammation [14].

A unifying factor that links diabetes mellitus types 1 and 2 (T1DM and T2DM, respectively) is the expression of vitamin D receptors (VDRs) in more than 30 biological tissues, including the pancreatic islet cells [15]. On the other hand, it is common for patients with T1DM and T2DM to have vitamin D deficiency [16]. Furthermore, several longitudinal and observational studies have demonstrated that low levels of serum 25-hydroxyvitamin D predict T2DM risk in Europeans [17], African-Americans [18], South Asians [18] and native American children [19]. Vitamin D correction through supplementation, therefore, may increase insulin secretion and improve glucose homeostasis; however, some studies have said that its effects on healthy individuals or in those with impaired glucose tolerance remain unclear [20]. Currently, there's scientific debate about how much vitamin D people need each day. The Institute of Medicine, in a long-awaited report released on November 30, 2010 recommends tripling the daily vitamin D intake for children and adults in the US and Canada, to 600 IU per day. [21] The report also recognized the safety of vitamin D by increasing the upper limit from 2000 to 4000 IU per day, and acknowledged that even at 4000 IU per day, there was no good evidence of harm. The new guidelines, however, are overly conservative about the recommended intake, and they do not give enough weight to some of the latest science on vitamin $\mathrm{D}$ and health. For bone health and chronic disease prevention, many people are likely to need more vitamin $\mathrm{D}$ than even these new government guidelines recommend.

Although there is no consensus on optimal serum levels of $25(\mathrm{OH}) \mathrm{D}$, vitamin D deficiency is defined by most experts as a level less than $20 \mathrm{ng} / \mathrm{mL}$ [22].

The purpose of this study was to examine the effect of vitamin D supplementation on mean serum values of HbA1c, the indicator for glycemic control and also on $25(\mathrm{OH})$ vitamin D concentrations in healthy and diabetic males and females. 


\section{Material \& Methods}

\subsection{Participants}

The study sample was (722) individuals which was selected out of 800 people from different areas in Damascus. Their age ranged from 36 to 61 years old. The sample consisted of 360 males and 362 females. The males and females were then divided into two groups; healthy and diabetic based on their diagnosis for at least 2 years history. Recruits granted consent to follow the instructions provided in this study and answered a questionnaire that contained several questions about their health status, medications taken, and when diagnosed if diabetic. Exclusion criteria consisted of a history of liver disease, renal failure, or cancer, or calcium or vitamin D supplement use or the presence of a parathyroid problem. For the diabetic group, none of the subjects reported any changes in their oral hypoglycemic agent as they followed their usual treatment regimen. The protocol of this study was approved by the council of Faculty of Health Sciences at University of Kalamoon. The study was carried out between June and September 2011. The participants were divided to the following groups as shown in Figure 1.

\subsection{Vitamin D Status}

Vitamin D nutritional status was assessed as deficient if $25(\mathrm{OH}) \mathrm{D}$ levels were $<20 \mathrm{ng} / \mathrm{mL}$, insufficient if between 20 and $30 \mathrm{ng} / \mathrm{mL}$, and sufficient if $\geq 30 \mathrm{ng} / \mathrm{mL}$ [21].

A five milliliter venous blood sample was obtained from each participant two times by qualified professional using standardized tubes. Each participant was given a number to keep results anonymous. Prior to blood sampling, all participants had been instructed to fast for at least eight hours and abstain from smoking. Blood samples were sent to Al Sham laboratory within two hours of blood draw where they underwent standardized (quality controlled) analyses. Serum 25(OH) D concentrations were measured by 25-OHD3 levels by ELISA assay using kit by IDS (USA). The glycosylated hemoglobin (HbAc1) was calculated using Direct Enzymatic Hemoglobin (HbA1c) Catalog Kit Number: BQ 004-EADD from BQ Kits, USA.

25-hydroxyvitamin D3 (25-OHD3), glycosylated hemoglobin A1c (HbA1c) values were measured in two occasions; at the start of the study and at the end. Vitamin D supplementation lasted for 90 days and each individual was given (2000 IU/day) of vitamin D tablets (Vigantoletten; Merck Pharma, Germany).

\subsection{Statistical Analysis}

Statistical analysis was done by using the Statistical Package for the Social Sciences (SSPS) for Windows version 16.5 (Chicago, Illinois) for evaluation of data. Repeated measures analysis of variance (ANOVA) was undertaken to compare values over time. A $P$-value $<0.05$ was deemed significant.

\section{Results}

The total number of the individuals participated in this study was 722 which consisted of 360 males (49.9) and

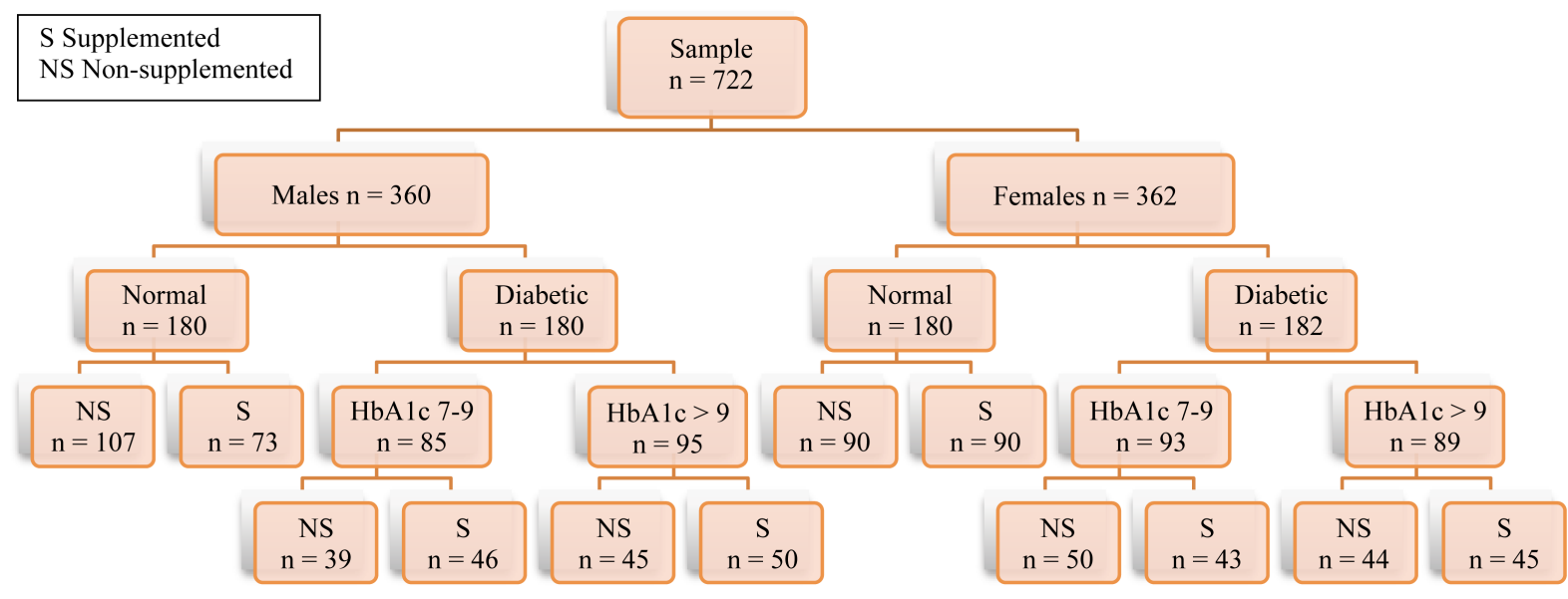

Figure 1. Sample distribution among groups. 
362 females (50.1\%). Their ages ranged from 36 and 61 years old. Males and females were also divided into two groups; healthy and diabetic. Both males and females in healthy group did not benefit from vitamin D supplementation as their serum HbA1c level did not change remarkably. In the healthy non-supplemented male group, HbA1c value changed from $6.85 \pm 1.43$ at the beginning of the experiment to $6.78 \pm 1.35$ and there was no significant difference $(P=0.06)$. The improvement percentage was $1 \%$. However, there was a slight decline in HbA1c value in the healthy male group when supplemented with vitamin D. HbA1c values dropped from $6.87 \pm$ 1.55 to $6.54 \pm 1.23$ before and after supplementation respectively (4.8\%) but the difference was not statistically significant too.

The females had also similar results in the healthy group; HbA1c in the non-supplemented females in the healthy group almost did not change. It was at the beginning of the study $6.98 \pm 1.34$ and became $6.93 \pm 1.32$ (0.72\%). Supplementation also did not lead to a significant change in HbA1c values in healthy females. Their HbA1c changed from $6.53 \pm 1.24$ to $6.34 \pm 1.11(2.9 \%)$ and the difference was not significant $(P=0.06)$. The results are shown in Table 1.

When compare healthy males and healthy females with regard to changes of their mean serum HbA1c values for non-supplemented and supplemented individuals, the study found that the highest improvement in HbA1c values $(4.8 \%)$ achieved among supplemented males followed by supplemented females (2.9\%), non-supplemented males (1\%) and finally non supplemented females $(0.72 \%)$ as shown in Figure 2.

Regarding males and females in the diabetic group, the effect of vitamin D supplementation was more apparent among the individuals with Hba1c values over 9 but also had a lowering effect with those who had HbA1c values 7 - 9. Mean serum HbA1c value for non-supplemented diabetic males in 7 - 9 group dropped slightly by only $5 \%$ from $8.54 \pm 2.32$ to $8.11 \pm 2.17$ and there was no significant difference $(P=0.05)$. In contrast, mean HbA1c values for males (for group 7 - 9) before supplementation were $8.21 \pm 2.54$ dropped to $7.11 \pm 2.02$ (13.4\%) and the difference was statistically significant $(P=0.03)$. The difference was more significant with the group of males who had HbA1c values over 9 because their HbA1c values plummeted from $10 \pm 2.98$ at the start of the study to $8.26 \pm 2.45$ after supplementation $(21.8 \%)$ and the difference was significant $(P=0.02)$ whereas non supplemented diabetic males of the same group, had a slight improvement in their mean serum HbA1c value which declined by only $2.7 \%$ from $10.92 \pm 3.22$ to $10.63 \pm 2.78$ and the difference was not significant $(P=0.06)$. The results are shown in Table 2.

Table 1. Mean serum HbA1c value changes in supplemented and non-supplemented healthy males and females.

\begin{tabular}{|c|c|c|c|c|c|c|c|c|}
\hline Group & \multicolumn{4}{|c|}{ Males n $=180$} & \multicolumn{4}{|c|}{ Females $n=182$} \\
\hline Subgroup & \multicolumn{2}{|c|}{$\mathrm{NS}=107$} & \multicolumn{2}{|c|}{$S=73$} & \multicolumn{2}{|c|}{$\mathrm{NS}=90$} & \multicolumn{2}{|c|}{$S=92$} \\
\hline $\begin{array}{l}\text { HbA1c Values at } \\
\text { beginning and end } \\
\text { of study }\end{array}$ & $6.85 \pm 1.43$ & $6.78 \pm 1.35$ & $6.87 \pm 1.55$ & $6.54 \pm 1.23$ & $6.98 \pm 1.34$ & $6.93 \pm 1.32$ & $6.53 \pm 1.24$ & $6.34 \pm 1.11$ \\
\hline $\begin{array}{l}\text { Percentage of } \\
\text { improvement }\end{array}$ & \multicolumn{2}{|c|}{1} & \multicolumn{2}{|c|}{4.8} & \multicolumn{2}{|c|}{0.72} & \multicolumn{2}{|c|}{2.9} \\
\hline$P$ value & \multicolumn{2}{|c|}{0.07} & \multicolumn{2}{|c|}{0.05} & \multicolumn{2}{|c|}{0.07} & \multicolumn{2}{|c|}{0.06} \\
\hline
\end{tabular}

NS = Non-supplemented; S = Supplemented; *' Significant difference $P<0.05$.

Table 2. Serum HbA1c value changes in supplemented and non-supplemented diabetic males.

\begin{tabular}{|c|c|c|c|c|c|c|c|c|}
\hline Group & \multicolumn{8}{|c|}{ Males $\mathrm{n}=180$} \\
\hline HbA1c value & \multicolumn{2}{|c|}{7 - 9 NS } & \multicolumn{2}{|c|}{7 - $9 \mathrm{~S}$} & \multicolumn{2}{|c|}{$9 \mathrm{NS}$} & \multicolumn{2}{|c|}{$9 \mathrm{~S}$} \\
\hline $\begin{array}{l}\text { Before and after } \\
\text { supplementation }\end{array}$ & $8.54 \pm 2.32$ & $8.11 \pm 2.17$ & $8.21 \pm 2.54$ & $7.11 \pm 2.02$ & $10.92 \pm 3.22$ & $10.63 \pm 2.78$ & $10.56 \pm 2.98$ & $8.26 \pm 2.45$ \\
\hline $\begin{array}{l}\text { Percentage of } \\
\text { improvement }\end{array}$ & \multicolumn{2}{|c|}{5.0} & \multicolumn{2}{|c|}{13.4} & \multicolumn{2}{|c|}{2.7} & \multicolumn{2}{|c|}{21.8} \\
\hline$P$ value & \multicolumn{2}{|c|}{0.05} & \multicolumn{2}{|c|}{$0.03^{*}$} & \multicolumn{2}{|c|}{0.06} & \multicolumn{2}{|c|}{$0.02^{*}$} \\
\hline
\end{tabular}

NS = Non-supplemented; $\mathrm{S}=$ Supplemented; ${ }^{*}$ Significant difference $P<0.05$. 


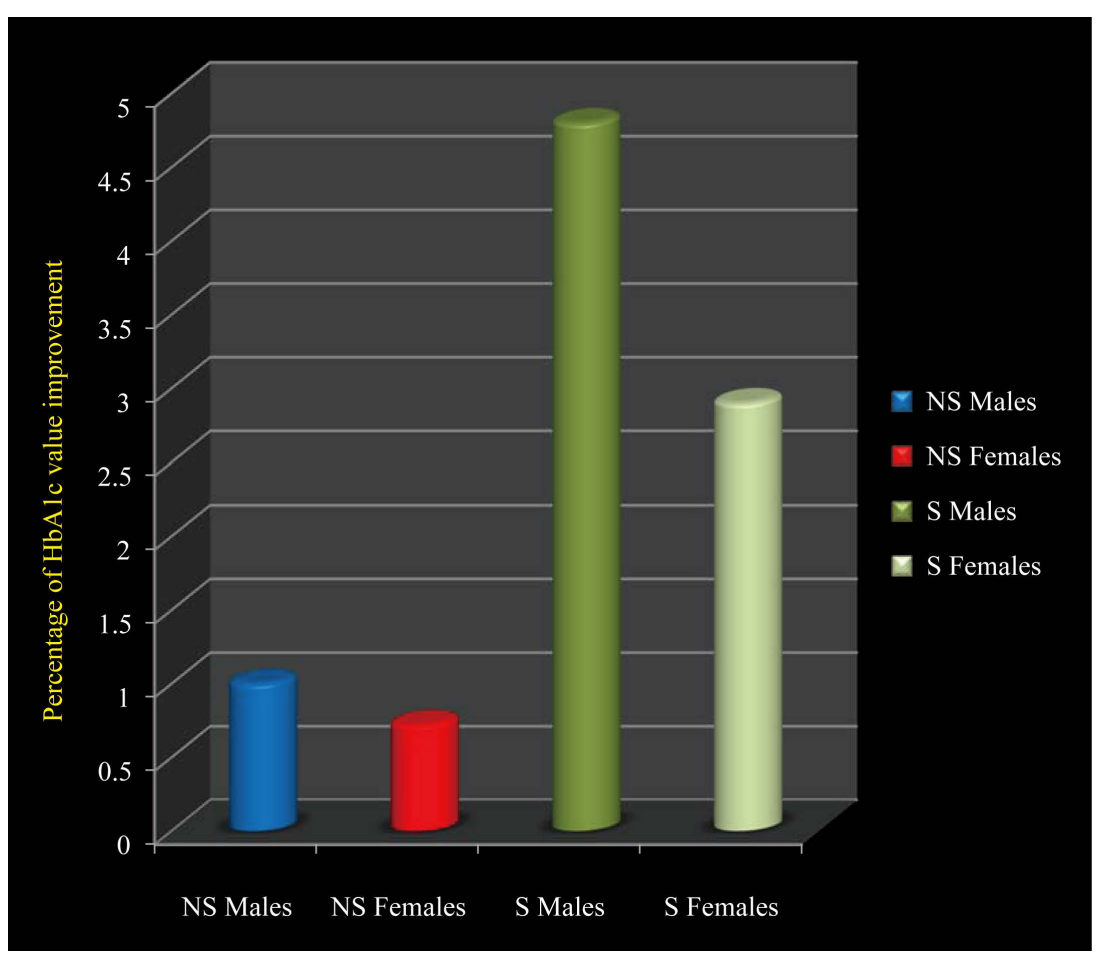

Figure 2. Comparison between supplemented and non-supplemented normal males and females.

The study had similar findings with regard to diabetic females. Mean serum HbA1c value for non-supplemented diabetic females (HbA1c 7 - 9 group) at the start of the study was $8.77 \pm 2.7$ dropped to $7.93 \pm 2.48$ $(9.6 \%)$ and the difference was not statistically significant $(P=0.05)$. Diabetic females who had HbA1c mean value 7 - 9 and given vitamin $D$ supplements showed better response. Their mean serum Hba1c value dropped from $8.42 \pm 2.81$ to $7.11 \pm 2.02(15 \%)$ and the difference was significant $(P=0.04)$.

Diabetic females who had HbA1c values over 9 showed better improvement with supplementation comparing with group HbA1c 7 - 9. Their mean serum HbA1c values plummeted from $10.96 \pm 3.21$ at the start of the study to $8.54 \pm 2.88(22.1 \%)$.The difference was statistically significant $(P=0.02)$ whereas non supplemented females for the same HbA1c group, showed a slight improvement (6.91\%) and the difference was not significant $(P=$ 0.05) as presented in Table 3.

The slight improvement in HbA1c values in non-supplemented diabetic individuals can be attributed to the hypoglycemic medications and their life style or by strict diabetes management.

A comparison was made between diabetic males and females to look at the effect of vitamin D supplementation on gender. The study found that maximum improvement in mean serum HbA1c values achieved among diabetic males and females who had HbA1c values over 9. Their mean HbA1c values were lowered by $21.8 \%$ and $22.1 \%$ for males and females respectively. Followed by diabetic females who were supplemented in (HbA1c 7 9 group) for an improvement of $15 \%$, and males in the same previous group by $13.4 \%$. Surprisingly, the highest improvement in HbA1c values can be seen among non-supplemented females in the group (HbA1c 7 - 9) by 9.61\% followed by non-supplemented diabetic females whose HbA1c value over 9 by 6.91 and non-supplemented diabetic males (HbA1c 7 - 9) and (HbA1c over 9) for 5\% and 2.7\% respectively. The comparison is presented in Figure 3.

This study has also looked at the changes in mean serum 25(OH) vitamin D concentrations in both non-supplemented and supplemented healthy and diabetic males and females. Data presented in Table 3 have shown that there was no significant difference in $25(\mathrm{OH})$ vitamin $\mathrm{D}$ in healthy non-supplemented males and females at the beginning and the end of the study. Mean serum $25(\mathrm{OH})$ vitamin $\mathrm{D}$ in healthy non supplemented males was $15.11 \pm 3.21 \mathrm{ng} / \mathrm{mL}$ dropped slightly to $14.78 \pm 2.43$ (2.9\%) and the difference was not statistically significant $(P=0.07)$. Similarly, in healthy non supplemented females, serum $25(\mathrm{OH})$ vitamin D concentration was 13.12 
Table 3. Serum HbA1c value changes in supplemented and non-supplemented diabetic females.

\begin{tabular}{|c|c|c|c|c|c|c|c|c|}
\hline Group & \multicolumn{8}{|c|}{ Females $n=182$} \\
\hline HbA1c value & \multicolumn{2}{|c|}{7 - 9 NS } & \multicolumn{2}{|c|}{$7-9 S$} & \multicolumn{2}{|c|}{$9 \mathrm{NS}$} & \multicolumn{2}{|c|}{$9 \mathrm{~S}$} \\
\hline $\begin{array}{l}\text { Before and after } \\
\text { supplementation }\end{array}$ & $8.77 \pm 2.7$ & $7.93 \pm 2.48$ & $8.42 \pm 2.81$ & $7.11 \pm 2.02$ & $10.85 \pm 2.78$ & $10.1 \pm 2.77$ & $10.96 \pm 3.21$ & $8.54 \pm 2.88$ \\
\hline $\begin{array}{l}\text { Percentage of } \\
\text { improvement }\end{array}$ & \multicolumn{2}{|c|}{9.6} & \multicolumn{2}{|c|}{15} & \multicolumn{2}{|c|}{6.91} & \multicolumn{2}{|c|}{22.1} \\
\hline$P$ value & \multicolumn{2}{|c|}{0.05} & \multicolumn{2}{|c|}{$0.04^{*}$} & \multicolumn{2}{|c|}{0.05} & \multicolumn{2}{|c|}{$0.02^{*}$} \\
\hline
\end{tabular}

NS = Non supplemented; $\mathrm{S}=$ Supplemented; ${ }^{*}$ Significant difference $P<0.05$.

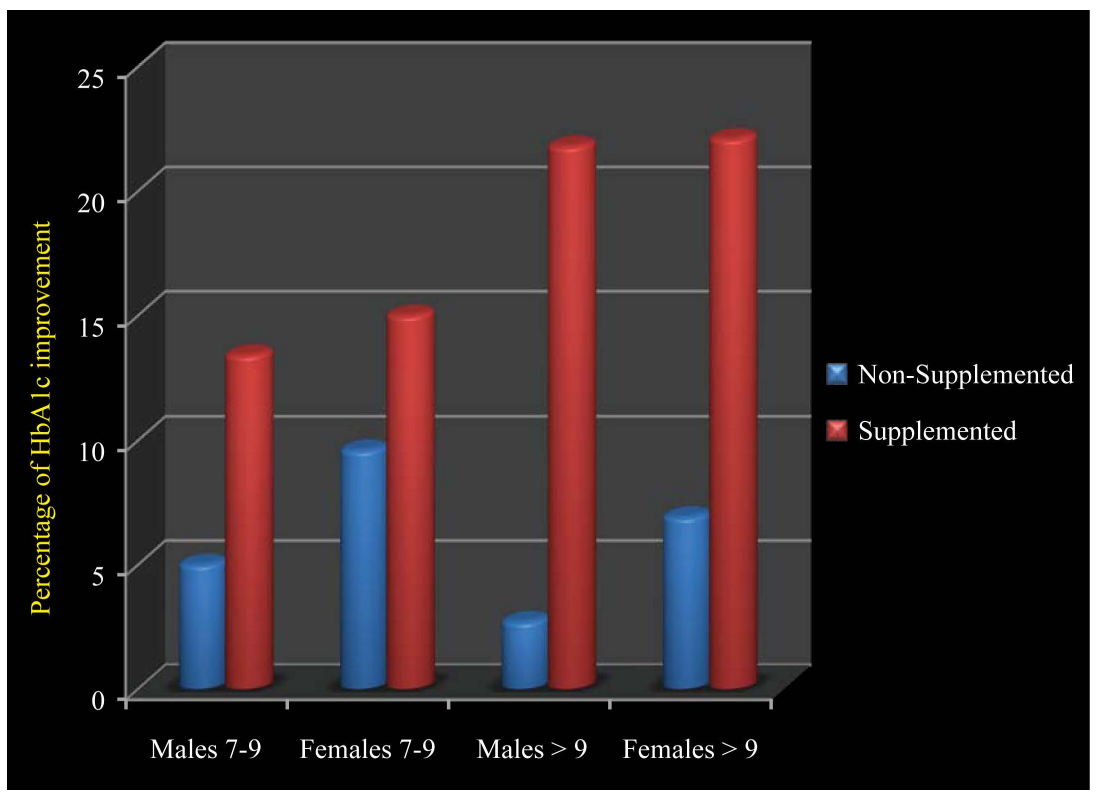

Figure 3. Comparison between supplemented and non-supplemented diabetic males and females.

\pm 12 improved to some extent to $13.56 \pm 3.33 \mathrm{ng} / \mathrm{mL}$ (3.4\%) and the difference was also not significant $(P=$ 0.06). Both groups are considered vitamin D deficient. Supplementation had a remarkable effect on serum $25(\mathrm{OH})$ vitamin D levels in both healthy males and females. Their mean serum 25(OH) vitamin D concentration before supplementation were $14.23 \pm 3.11$ and $14.54 \pm 3.54 \mathrm{ng} / \mathrm{mL}$ changed to $23.89 \pm 5.54$ and $21.47 \pm 4.76$ $\mathrm{ng} / \mathrm{mL}$ after supplementation (67.9\%) and (47.7\%) for both males and females $(P=0.001$ and $P=0.002)$ respectively. It's worth mentioning that supplementation upgraded the status of the individuals in this group from vitamin D deficient to vitamin D sufficient which will have an impact on their nutritional and health status. The data are shown in Table 4 and Figure 4.

This study has also examined the effect of supplementation on males and females in the diabetic group. Table 5 has shown that serum $25(\mathrm{OH})$ vitamin D concentrations changed in supplemented males and females in the diabetic group at the beginning and the end of the study.

Supplementation with vitamin D had dramatically improved mean serum 25(OH) vitamin D levels in diabetic group. In males with HbA1c values between 7 - 9, mean serum 25(OH) vitamin D concentration was $13.11 \pm$ 3.65 before supplementation changed to $20.06 \pm 2.56$ after supplementation (53\%) and the difference was significant $(P=0.02)$. Males who had higher HbA1c values (more than 9 ) also benefited from vitamin $\mathrm{D}$ supplementation. Their mean serum 25(OH) vitamin D changed from $12.83 \pm 2.14$ to $20.09 \pm 5.12 \mathrm{ng} / \mathrm{mL}(65.6 \%)(P$ $=0.01$. .

The same thing applies to diabetic females. Females with $25(\mathrm{OH})$ vitamin D values $7-9$, their mean serum $25(\mathrm{OH})$ vitamin D changed from $14.62 \pm 3.76$ to $19.26 \pm 2.25(31.7 \%)(P=0.03)$ and for females with HbA1c 
Table 4. Mean serum 25(OH) vitamin D concentration in healthy group.

\begin{tabular}{|c|c|c|c|c|c|c|c|c|}
\hline Group & \multicolumn{4}{|c|}{ Males } & \multicolumn{4}{|c|}{ Females } \\
\hline $25(\mathrm{OH})$ vit $\mathrm{D}$ & \multicolumn{2}{|c|}{ NS } & \multicolumn{2}{|c|}{$\mathrm{S}$} & \multicolumn{2}{|c|}{ NS } & \multicolumn{2}{|c|}{$\mathrm{S}$} \\
\hline Before and after & $15.11 \pm 3.21$ & $14.78 \pm 2.43$ & $14.23 \pm 3.11$ & $23.89 \pm 5.54$ & $13.12 \pm 3.01$ & $13.56 \pm 3.33$ & $14.54 \pm 3.54$ & $21.47 \pm 4.76$ \\
\hline $\begin{array}{l}\text { Percentage of } \\
\text { improvement }\end{array}$ & \multicolumn{2}{|c|}{2.9} & \multicolumn{2}{|c|}{67.9} & \multicolumn{2}{|c|}{3.4} & \multicolumn{2}{|c|}{47.7} \\
\hline$P$ value & \multicolumn{2}{|c|}{0.07} & \multicolumn{2}{|c|}{$0.001^{*}$} & \multicolumn{2}{|c|}{0.06} & \multicolumn{2}{|c|}{$0.002^{*}$} \\
\hline
\end{tabular}

$\mathrm{S}=$ Supplemented; NS = Non Supplemented; ${ }^{*}$ Significant difference $(P<0.05)$.

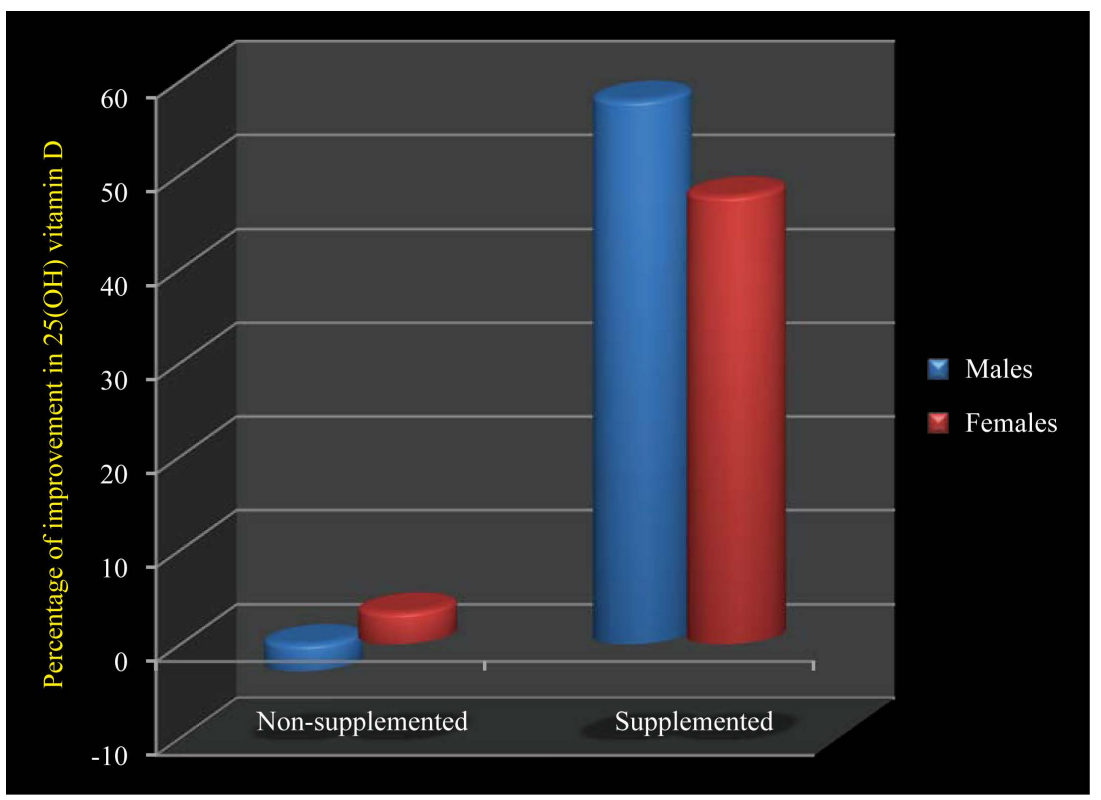

Figure 4. Comparison between healthy non-supplemented and supplemented males and females.

over 9, changed from $11.32 \pm 3.54$ to $20.06 \pm 5.11 \mathrm{ng} / \mathrm{mL}(77.2 \%)(P=0.01)$. These results are shown in Table 5 and Figure 5.

This study has also compared the effect of vitamin D supplementation on mean serum 25(OH) vitamin D concentration between healthy and diabetic males and females.

The highest improvement value of mean serum $25(\mathrm{OH})$ vitamin $\mathrm{D}$ has been found among supplemented diabetic females who had HbA1c > 9 with an improvement percentage $77.2 \%$ followed by supplemented diabetic males in the same group (56.6\%). Surprisingly, diabetic males with HbA1c 7 - 9 did better than their females counterparts (53\%) and $31.7 \%$ respectively. Healthy males also benefited from vitamin D supplementation. Their $25(\mathrm{OH})$ vitamin D improved by $67.9 \%$ followed by supplemented diabetic males and females by $53 \%$ and $31.7 \%$ respectively. The improvement in $25(\mathrm{OH})$ vitamin $\mathrm{D}$ in healthy group was very obvious in males $67.9 \%$ followed by females $47.7 \%$. Without supplementation, healthy females had $3.4 \%$ improvement in $25(\mathrm{OH})$ vitamin D level whereas males in the same group had decrease in their $25(\mathrm{OH})$ vitamin D level as shown in Figure 6.

\section{Discussion}

The findings of this study indicate that the metabolic profile of T2DM subjects, particularly females, is significantly improved over after the onset of vitamin D3 supplementation, suggesting that vitamin D correction is a promising a tool in glycemic control in vitamin D-deficient populations. The gender effect can be attributed indirectly to either difference in hormone secretion and/or target tissue effects and body fat distribution, both of 
Table 5. Changes in mean serum 25(OH) vitamin D concentration in the diabetic group after supplementation.

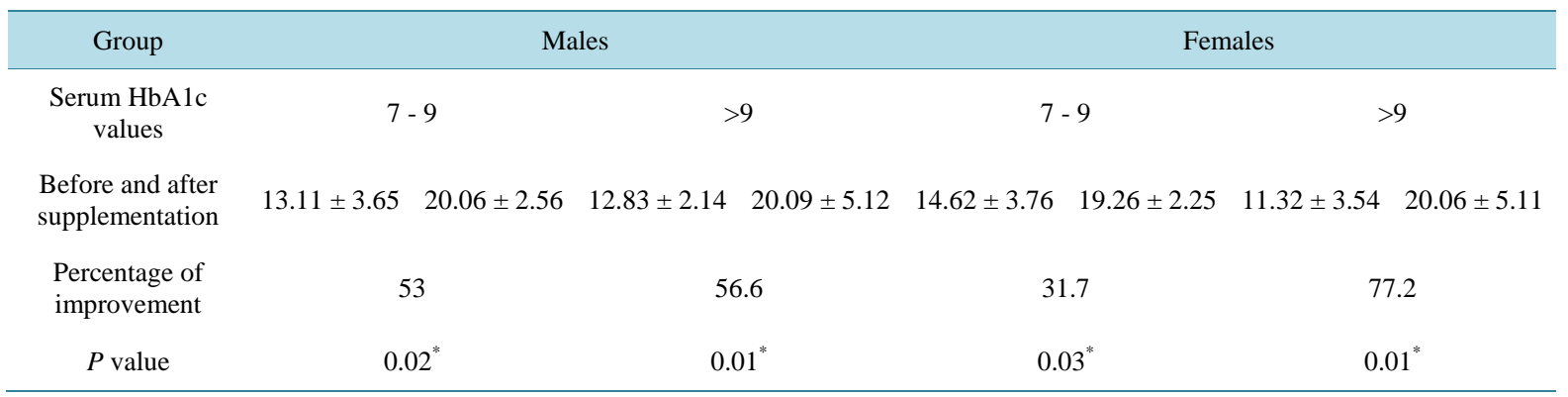

"Significant difference $(P<0.05)$.

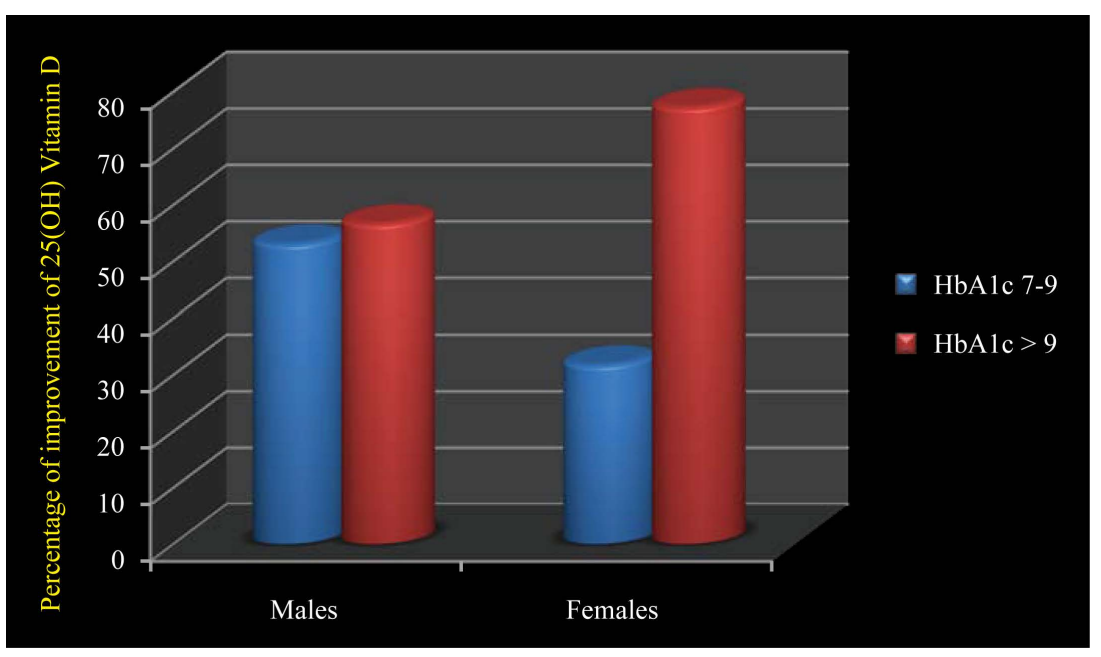

Figure 5. Comparison between supplemented diabetic males and females according to their HbA1c values.

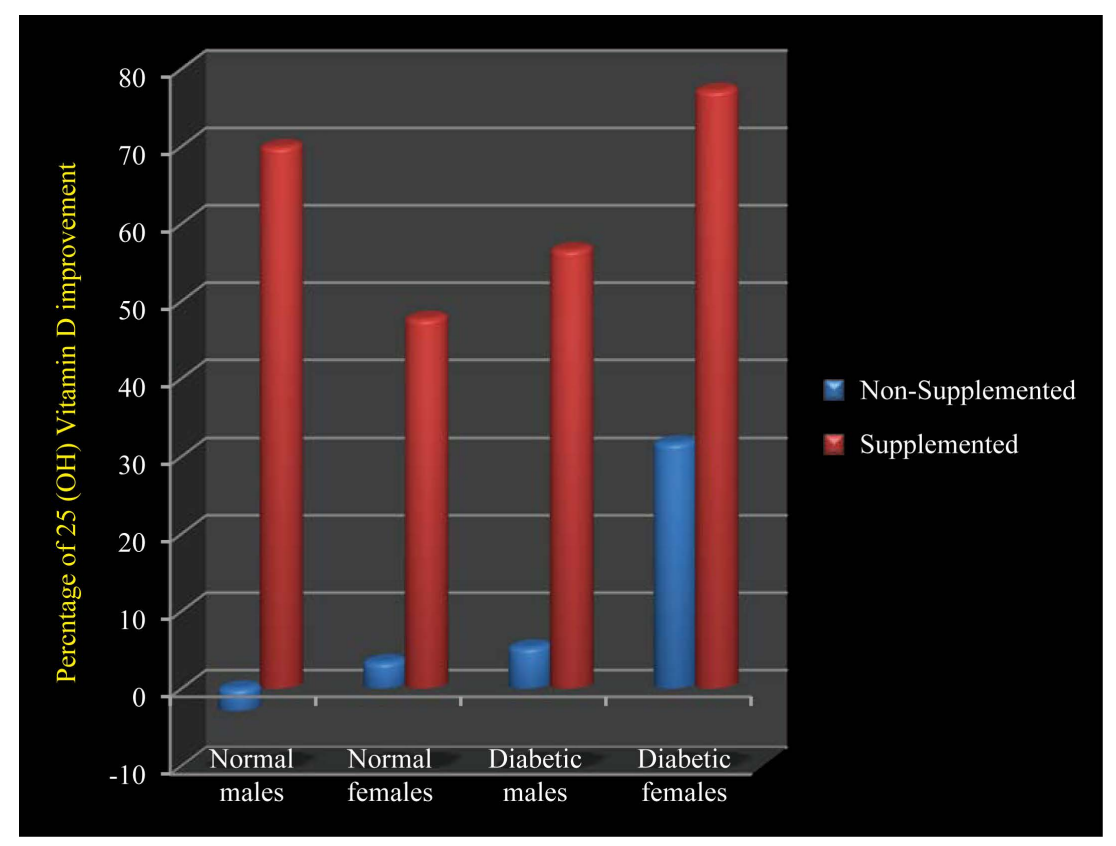

Figure 6. Comparison between supplemented and non-supplemented diabetic males and females. 
which can contribute to degrees of insulin resistance. In contrast, some studies failed to show an improvement in levels of blood sugar, glucose tolerance or insulin sensitivity following vitamin D supplement in both diabetic and non-diabetic subjects. Conflicting results on metabolism of glucose and insulin has been observed by administration of different forms of vitamin D such as cholecalciferol, orgocalciferol, and active form of this vita$\min [23]$.

In another study with 32 diabetic patients under metformin and bedtime insulin treatment, consumption of 40,000 IU weekly cholecalciferol for 6 months had no effect on fasting blood sugar, insulin, c-peptide and HbA1c compared with placebo group [21]. One assumption could be that since insulin secretion is a calcium dependent process, it can indirectly be influenced by effect of vitamin D on calcium absorption. Although a single injection of vitamin D caused significant changes in serum levels of vitamin $\mathrm{D}$, the insulin resistant status has become worsen.

Based on available clinical and epidemiological data, the positive effects of vitamin D seem to be primarily related to its action on insulin secretion and sensitivity and secondary to its action on inflammation. Future studies specifically designed to investigate the role of vitamin $\mathrm{D}$ on type 2 diabetes using inflammation as the main outcome are urgently needed in order to provide a more robust link between vitamin D, inflammation and type 2 diabetes [24].

Experimental evidence also supported the idea that vitamin D may play a role in the pathogenesis of DM [25]. Additionally, it has been reported that vitamin D supplementation may decrease the risk of developing DM in long term period. The protective effects of vitamin D are mediated through the immune system and calcium metabolism. Vitamin D may also have a direct effect on pancreatic beta cells [26] result in an improvement in glycemic control [27].

\section{Conclusion}

Supplementation with vitamin D has improved HbA1c values in both healthy and diabetic males and females. But the improvement was more conspicuous and statistically significant in the diabetic group especially with those who had worse control of their glucose level (HbA1c values over 9). Also supplementation increased mean serum 25(OH) vitamin D concentrations in males and females upgrading their status from deficient to sufficient groups which will have positive impact on their nutritional and health status. Moreover further studies are needed to show the definite effect of injection of vitamin D on control of diabetes and its risk.

\section{References}

[1] International Diabetes Federation (IDF) (2011) Diabetes Atlas Global Burden, Epidemiology and Morbidity. Diabetes and Impaired Glucose Tolerance. http://www.diabetesaltas.org/content/diabetes-and-impaired-glucose-tolerance

[2] Hyppönen, E., Läärä, E., Reunanen, A., Järvelin, M. and Virtanen, S. (2001) Intake of Vitamin D and Risk of Type 1 Diabetes: A Birth-Cohort Study. Lancet, 358, 1500-1503.

[3] James, W.P.T. (2008) 22nd Marabou Symposium: The Changing Faces of Vitamin D. Nutrition Reviews, 66, $286-290$. http://dx.doi.org/10.1111/j.1753-4887.2008.00034.x

[4] Lips, P. (2007) Vitamin D Status and Nutrition in Europe and Asia. The Journal of Steroid Biochemistry and Molecular Biology, 103, 620-625. http://dx.doi.org/10.1016/j.jsbmb.2006.12.076

[5] Parfitt, A.M., Gallagher, J.C., Heaney, R.P., Johnston, C.C., Neer, R. and Whedon, G.D. (1982) Vitamin D and Bone Health in the Elderly. The American Journal of Clinical Nutrition, 36, 1014-1031.

[6] Peacoak, M. (1998) Effect of Calcium and Vitamin D Insufficiency on the Skeleton. Osteoporosis International, 8, S45-S51. http://dx.doi.org/10.1007/PL00022733

[7] Deluca, H.F. (1988) The Vitamin D Story: A Collaborative Effort of Basic Science and Clinical Medicine. The FASEB Journal, 2, 224-236.

[8] Bouillon, R. (2001) Vitamin D: Photosynthesis, Metabolism, and Action to Clinical Applications. In: Degroot, L., Jameson, J.L. and Burger, H.G., Eds., Endocrinology, 3rd Edition, WB Saunders, Philadelphia, 1009-1028.

[9] Bouillon, R., Carmeliet, G., Daci, E., et al. (1998) Vitamin D Metabolism and Action. Osteoporosis International, 8, S13-S19. http://dx.doi.org/10.1007/PL00022727

[10] Holick Michael, F. (2010) The Vitamin D Deficiency Pandemic: A Forgotten Hormone Important for Health. Public Health Reviews, 32, 267-283.

[11] Wang, H., Xia, N., Yang, Y. and Peng, D.-Q. (2012) Influence of Vitamin D Supplementation on Plasma Lipid Profiles: 
A Meta-Analysis of Randomized Controlled Trials. Lipids in Health and Disease, 11, 42.

[12] Alvarez, J.A. and Ashraf, A. (2010) Role of Vitamin D in Insulin Secretion and Insulin Sensitivity for Glucose Homeostasis. International Journal of Endocrinology, 2010, Article ID: 351385.

[13] Afsaneh, T., Mahnaz, M. and Zahra, A. (2013) The Effect of Vitamin D on Insulin Resistance in Patients with Type 2 Diabetes. Diabetology \& Metabolic Syndrome, 5, 8.

[14] Muszkat, P., Camargo, M.B.R., Griz, L.H.M. and Lazaretti-Castro, M. (2010) Evidence-Based Non-Skeletal Actions of Vitamin D. Arquivos Brasileiros de Endocrinologia \& Metabologia, 54, 110-117. http://dx.doi.org/10.1590/S0004-27302010000200005

[15] Attila, C., Knekt, P., Männistö, S., Rissanen, H., Laaksonen, M.A., Montonen, J. and Reunanen, A. (2007) Serum 25-Hydroxyvitamin D Concentration and Subsequent Risk Of type 2 Diabetes. Diabetes Care, 30, 2569-2570. http://dx.doi.org/10.2337/dc07-0292

[16] Al-Daghri, N.M., Alkharfy, K.M., Al-Othman, A., El-Kholie, E., Moharram, O., Alokail, M.S., Al-Saleh, Y., Sabico, S., Kumar, S. and Chrousos, G.P. (2012) Vitamin D Supplementation as an Adjuvant Therapy for Patients with T2DM: An 18-Month Prospective Interventional Study. Cardiovascular Diabetology, 11, 85.

[17] Harris, S.S. (2011) Does Vitamin D Deficiency Contribute to Increased Rates of Cardiovascular Disease and Type 2 Diabetes in African Americans. The American Journal of Clinical Nutrition, 93, 1175S-1178S. http://dx.doi.org/10.3945/ajcn.110.003491

[18] Boucher, B.J. (2006) Hypovitaminosis D and Risk of Type 2 Diabetes in British South Asians. Diabetic Medicine, 23, 336. http://dx.doi.org/10.1111/j.1464-5491.2006.01825a.x

[19] Nsiah-Kumi, P.A., Erickson, J.M., Beals, J.L., Ogle, E.A., Whiting, M., Brushbreaker, C., Borgeson, C.D., Qiu, F., Yu, F. and Larsen, J.L. (2012) Vitamin D Insufficiency Is Associated with Diabetes Risk in Native American Children. Clinical Pediatrics (Phila), 51, 146-153. http://dx.doi.org/10.1177/0009922811417290

[20] Heshmat, R., Tabatabaei-Malazy, O., Abbaszadeh-Ahranjani, S., Shahbazi, S., Khooshehchin, G., Bandarian, F. and Larijani B. (2012) Effect of Vitamin D on Insulin Resistance and Anthropometric Parameters in Type 2 Diabetes; a Randomized Double-Blind Clinical Trial. DARU Journal of Pharmaceutical Sciences, 20, 10. http://dx.doi.org/10.1186/2008-2231-20-10

[21] Institute of Medicine (2010) Dietary Reference Intakes for Calcium and Vitamin D. National Academies Press, Washington DC.

[22] Holick, M.F. (2007) Vitamin D Deficiency. The New England Journal of Medicine, 357, 266-281. http://dx.doi.org/10.1056/NEJMra070553

[23] Jorde, R. and Figenschau, Y. (2009) Supplementation with Cholecalciferol Does Not Improve Glycaemic Control in diabetic Subjects with Healthy Serum 25-Hydroxyvitamin D Levels. European Journal of Nutrition, 48, 349-354. http://dx.doi.org/10.1007/s00394-009-0020-3

[24] Chagas, C.E.A., Borges, M.C., Martini, L.A. and Rogero, M.M. (2012) Focus on Vitamin D, Inflammation and Type 2 Diabetes. Nutrients, 4, 52-67.

[25] Pittas, A.G., Sun, Q., Manson, J.E., Dawson-Hughes, B. and Hu, F.B. (2010) Plasma 25-Hydroxyvitamin D Concentration and Risk of Incident Type 2 Diabetes in Women. Diabetes Care, 33, 2021-2023. http://dx.doi.org/10.2337/dc10-0790

[26] İyidir, O.T. and Altınova, A.E. (2012) Vitamin D and Diabetes Mellitus. Turkish Journal of Endocrinology and Metabolism, 16, 89-94.

[27] Schwalfenberg, G. (2008) Vitamin D and Diabetes: Improvement of Glycemic Control with Vitamin D3 Repletion. Canadian Family Physician, 54, 864-866. 